Article

\title{
Tailoring Properties of Hafnium Nitride Thin Film via Reactive Gas-Timing RF Magnetron Sputtering for Surface Enhanced-Raman Scattering Substrates
}

Nguentra Sucheewa ${ }^{1}$, Winadda Wongwiriyapan ${ }^{1, *}$, Annop Klamchuen ${ }^{2, *} \mathbb{D}$, Michiko Obata ${ }^{3}$, Masatsugu Fujishige ${ }^{4}$, Kenji Takeuchi ${ }^{3,5}$, Tossaporn Lertvanithphol ${ }^{6}$, Tuksadon Wutikhun ${ }^{2}$, Saifon Kullyakool ${ }^{2}$, Wanwalee Auttasiri ${ }^{7}$, Nataporn Sowasod ${ }^{7}$, Theerayut Prataen ${ }^{7}$, Wiwut Tanthapanichakoon ${ }^{8}$ and Jiti Nukeaw ${ }^{1}$

check for

updates

Citation: Sucheewa, N.;

Wongwiriyapan, W.; Klamchuen, A.;

Obata, M.; Fujishige, M.; Takeuchi, K.;

Lertvanithphol, T.; Wutikhun, T.;

Kullyakool, S.; Auttasiri, W.; et al.

Tailoring Properties of Hafnium

Nitride Thin Film via Reactive

Gas-Timing RF Magnetron

Sputtering for Surface Enhanced-

Raman Scattering Substrates. Crystals

2022, 12, 78. https://doi.org/

$10.3390 /$ cryst 12010078

Academic Editor: Alessandro

Chiasera

Received: 26 November 2021

Accepted: 2 January 2022

Published: 6 January 2022

Publisher's Note: MDPI stays neutral with regard to jurisdictional claims in published maps and institutional affiliations.

Copyright: () 2022 by the authors Licensee MDPI, Basel, Switzerland. This article is an open access article distributed under the terms and conditions of the Creative Commons Attribution (CC BY) license (https:// creativecommons.org/licenses/by/ $4.0 /)$.
1 College of Materials Innovation and Technology, King Mongkut's Institute of Technology Ladkrabang, Chalongkrung Rd., Ladkrabang, Bangkok 10520, Thailand; 60607008@kmitl.ac.th (N.S.); jiti.nu@kmitl.ac.th (J.N.)

2 National Nanotechnology Center (NANOTEC), National Science and Technology Development Agency (NSTDA), Pathum Thani 12120, Thailand; Tuksadon@nanotec.or.th (T.W.); Saifon.kul@ncr.nstda.or.th (S.K.)

3 Interdisciplinary Cluster for Cutting Edge Research, Research Initiative for Supra-Materials, Shinshu University, 4-17-1 Wakasato, Nagano 380-8553, Japan; obatam@shinshu-u.ac.jp (M.O.); takeuchi@endomoribu.shinshu-u.ac.jp (K.T.)

4 Global Aqua Innovation Center, Shinshu University, 4-17-1 Wakasato, Nagano 380-8553, Japan; fshige@shinshu-u.ac.jp

5 Department of Water Environment and Civil Engineering, Faculty of Engineering, Shinshu University, 4-17-1 Wakasato, Nagano 380-8553, Japan

6 National Electronics and Computer Technology Center (NECTEC), National Science and Technology Development Agency (NSTDA), Pathum Thani 12120, Thailand; Tossaporn.ler@nectec.or.th

7 Division of Chemical Process Engineering Technology, Faculty of Engineering and Technology, King Mongkut's University of Technology North Bangkok (Rayong Campus), Rayong 21120, Thailand; s6012011610043@email.kmutnb.ac.th (W.A.); nataporn.s@eat.kmutnb.ac.th (N.S.); s5912011620212@email.kmutnb.ac.th (T.P.)

8 Academy of Science, Royal Society of Thailand, Sanam Sua Pa, Dusit District, Bangkok 10300, Thailand; wiwut@yahoo.com

* Correspondence: winadda.wo@kmitl.ac.th (W.W.); annop@nanotec.or.th (A.K.)

Abstract: This study successfully demonstrated the tailoring properties of hafnium nitride (HfN) thin films via reactive gas-timing (RGT) RF magnetron sputtering for surface-enhanced Raman spectroscopy (SERS) substrate applications. The optimal RGT sputtering condition was investigated by varying the duration time of the argon and nitrogen gas sequence. The RGT technique formed thin films with a grain size of approximately $15 \mathrm{~nm}$. Additionally, the atomic ratios of nitrogen and hafnium can be controlled between 0.24 and 0.28 , which is greater than the conventional technique, resulting in a high absorbance in the long wavelength region. Moreover, the HfN thin film exhibited a high Raman signal intensity with an EF of $8.5 \times 10^{4}$ to methylene blue molecules and was capable of being reused five times. A superior performance of HfN as a SERS substrate can be attributed to its tailored grain size and chemical composition, which results in an increase in the hot spot effect. These results demonstrate that the RGT technique is a viable method for fabricating HfN thin films with controlled properties at room temperature, which makes them an attractive material for SERS and other plasmonic applications.

Keywords: hafnium nitride; reactive gas timing; sputtering; surface-enhanced Raman spectroscopy

\section{Introduction}

Surface-enhanced Raman spectroscopy (SERS) is a powerful vibrational spectroscopy technique that is characterized by its rapid, nondestructive, ultrasensitive, and fingerprint 
diagnostic capabilities. As a result, it has a broad range of promising applications in chemical and biological analysis at trace levels [1,2]. In comparison with conventional Raman spectroscopy, the primary result of SERS is a dramatically enhanced Raman intensity of several orders of magnitude, which is commonly agreed to be the result of the combined contribution of electromagnetic and chemical mechanisms. The chemical mechanism covers effects caused by molecular adsorptions that result in the change of the electron cloud during molecular vibration via chemical complexation, charge transfer, or charge transfer resonance [3,4]. In contrast, the electromagnetic mechanism is based on the plasmonic oscillation resonance of conducting electrons in a roughened surface, which amplifies the incident light wave and hence increases the Raman scattering intensity of the analyst molecule [5]. Normally, SERS substrates are made of noble metal, such as gold, silver, and copper, due to localized surface plasmon resonance (LSPR) inducing highly electromagnetic fields in the nanostructures. However, the applications of the SERS metal substrate are extremely limited due to its high cost, low stability, and mechanical rupture [6,7]. Obtaining a stable and reusable SERS substrate using a noble metal nanostructure remains a serious challenge.

Recently, transition metal nitride (TMN) has received attention as an alternative plasmonic material with significant potential in the visible to near-infrared ranges of an excitation light [8], comparable to that of traditional noble metals. TMNs (e.g., titanium nitride (TiN), zirconium nitride $(\mathrm{ZrN})$, and hafnium nitride $(\mathrm{HfN}))$ are particularly interesting due to their high melting point, strong mechanical properties, intrinsic chemical stability in harsh environments, and localized plasmon resonances that are extremely comparable to gold [9-12]. Recent research has demonstrated that $\mathrm{HfN}$ exhibits exceptional quality factors for surface plasmon polaritons (SPPs) and LSPR [10,13], which are the real and imaginary components of the dielectric function. Additionally, the HfN material has excellent corrosion resistance and thermal and chemical stability. Thus, plasmonic materials based on HfN are appropriate for SERS applications.

An extensive study on HfN thin films is aimed at controlling the stoichiometry, which is critical in determining the optical properties [11]. To form the films, physical vapor deposition (PVD) techniques, such as magnetron sputtering or pulsed laser deposition, are generally used. Although several research groups have demonstrated the fabrication of HfN thin films with controllable surface morphology and chemical composition, external energy sources, such as substrate bias voltage [14], substrate heating during growth [15], post annealing [16], and ionized magnetron sputter deposition [17], must be included. Externally introduced procedures complicate and increase the expense of the operation, and they are not accessible for fabricating an $\mathrm{HfN}$ film at room temperature. Among PVD techniques, the reactive gas-timing (RGT) RF magnetron sputtering approach is one that allows for adjusting the amount of sputtered atoms and enriching energy formation at room temperature [18-21].

Here, we demonstrate the tailored properties of HfN thin films through RGT RF magnetron sputtering. The optimal RGT sputtering condition was investigated by varying the duration time of the argon and nitrogen gas sequence. It was found that the RGT technique formed thin films with a grain size of approximately $15 \mathrm{~nm}$, which is smaller than the grain size formed by the conventional mixture gases technique. The fcc-HfN was formed, and the crystallinity increased with a longer nitrogen duration time. Additionally, the atomic ratios of nitrogen and hafnium can be controlled between 0.24 and 0.28 , which is greater than the conventional technique, resulting in a high absorbance in the long wavelength region. Moreover, the HfN thin film prepared by the RGT technique can be utilized as a reusable SERS substrate.

\section{Experimental Section}

\subsection{Deposition of HfN Thin Film}

HfN thin films were fabricated on $\mathrm{Si}(111)$ and glass slide substrates using RGT RF magnetron sputtering (UNIVAX380, Leybold Singapore Pte. Ltd., Singapore). A 2-inch 
diameter with a 0.25 -inch thick hafnium target (99.99\%) was used as the sputtering target. The Si(111) and glass slide substrates were cleaned via an alcohol process, in which Si(111) substrates were ultrasonically cleaned with acetone, ethanol, and deionized water for $10 \mathrm{~min}$, respectively, and dried with the nitrogen flow. The samples were then transferred to the high vacuum chamber for thin film deposition. The distance between the target and the substrate was set at $70 \mathrm{~mm}$. The substrates were mounted on a rotational holder substrate, which was driven by a motor at a rotation speed of $10 \mathrm{rpm}$. High-purity argon and nitrogen (99.9999\%) were supplied as sputtering gas. When a base pressure reached $4.6 \times 10^{-6}$ mbar, a constant flow of argon and nitrogen gas was introduced to the chamber at 30 and $2 \mathrm{sccm}$, respectively. The RF power and working pressure for sputtering were 150 watts and $7 \times 10^{-3}$ mbar, respectively.

During the film deposition, the RGT technique was utilized to fabricate HfN thin films by controlling the on-off sequences of argon and nitrogen gases at specific temporal intervals in order to operate alternate gas flows into the vacuum chamber by programmable mass flow controllers, as shown in Figure 1. In this work, turn-on sequences of nitrogen gas was changed from 1 to $7 \mathrm{~s}$ and switched to turn-on sequences of argon fixing at $10 \mathrm{~s}$. In other words, the duration time of the argon and nitrogen gas sequence was by a factor of 10:1, 10:3, 10:5, and 10:7 (hereinafter referred to as RGT1, RGT3, RGT5, and RGT7, respectively). For comparison, an HfN thin film was also prepared by a conventional mixture gases technique (a mixture of argon $30 \mathrm{sccm}$ and nitrogen $2 \mathrm{sccm}$ ) (hereinafter referred to as MIX). It should be noted that the deposition processes were carried out at room temperature (i.e., the substrate was not heated during or after deposition). The thickness of HfN thin films was approximately $100 \mathrm{~nm}$, which was measured by a thickness monitor (Inficon SQM-160, Cologne, Germany). Details of all the conditions are summarized in Supplementary Materials Table S1.

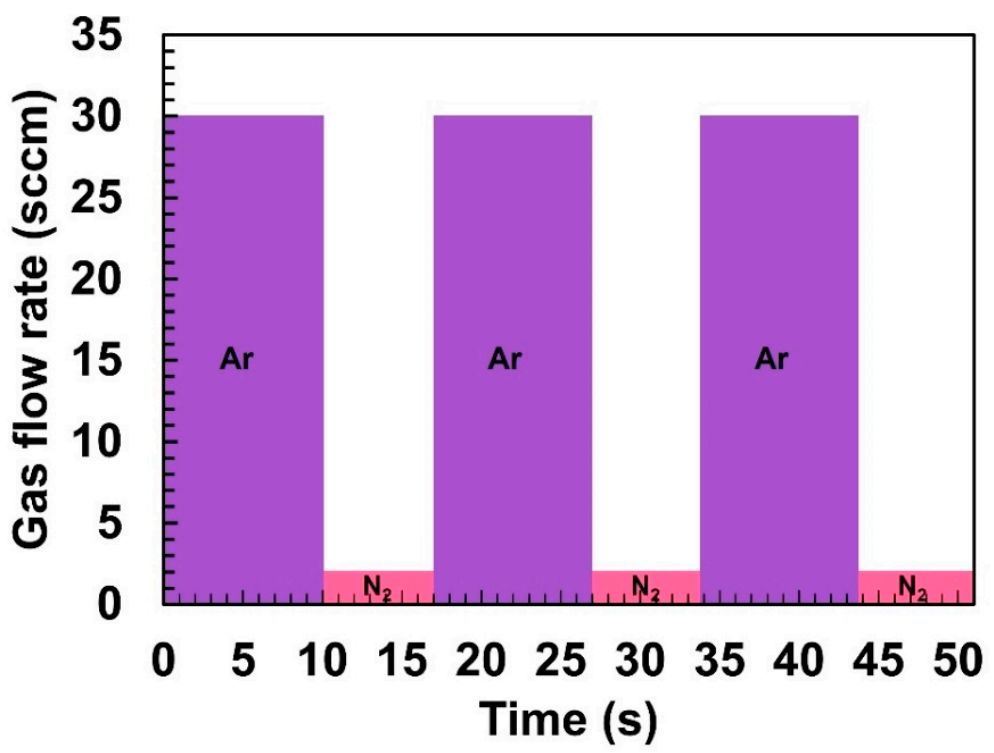

Figure 1. Schematic diagram of gas sequence during RGT RF magnetron technique by programmable mass flow controllers.

\subsection{Characterization of HfN Thin Film}

The crystal structure of HfN thin films was examined by an X-ray diffractometer (XRD, Bruker D8 Advance, San Jose, CA, USA) with a $2 \theta$ scan range of 30-80 degrees and a sampling pitch of 0.02 degree. The thickness of HfN thin films was determined from a cross-sectional image measured by field-emission scanning electron microscope (FE-SEM, FEI, VERSA 3D, Apeldoorn, The Netherlands) with an accelerated voltage of $20 \mathrm{kV}$. An atomic force microscope (AFM, Seiko SPA400, Chiba, Japan) was employed to investigate the morphology of HfN thin films in tapping mode using a Si cantilever with a radius of curvature less than $10 \mathrm{~nm}$. The Raman signal of HfN thin films was measured by confocal Raman spectroscopy (NT-MDT NTEGRA Spectra, Moscow, Russia) 
with an excitation wavelength of $632 \mathrm{~nm}$. The chemical composition was analyzed via X-ray photoelectron spectroscopy (XPS, ULVAC-PHI, PHI Quantera II, Chigasaki, Japan) with nonmonochromatic Al-K $\alpha$ X-ray source operating at $25 \mathrm{~W}$. The deconvolution of the XPS spectrum was performed through the Shirley background and the GL(30) function (Gauss and Lorentzian functions at a ratio of 30:70) [22]. The parameters for peak fitting are based on a full width at half maximum of about $1.2 \mathrm{eV}$ [22].

Finally, the SERS performance using HfN thin films fabricated via RGT RF magnetron sputtering technique was investigated. Methylene blue (MB), a Raman active molecule, was prepared at a concentration range of $10^{-3}$ to $10^{-6} \mathrm{M}$ for testing SERS activity. A microscope with a $100 \times$ objective lens, a laser beam with an excitation wavelength of $632 \mathrm{~nm}$, and a charge-coupled device (CCD) with a resolution of $4 \mathrm{~cm}^{-1}$ were employed to record the SERS spectra. The Raman enhancement factor $(E F)$ is expressed as:

$$
E F=\frac{I_{\text {SERS }}}{I_{\text {ref }}} \times \frac{N_{\text {ref }}}{N_{\text {SERS }}}
$$

where $I_{\text {SERS }}$ is the enhanced intensity of adsorbed MB molecules on the SERS substrate, $I_{\text {ref }}$ is the spontaneous Raman scattering intensity from the bulk MB molecules under the laser spot on the bare Si substrate, $N_{\text {ref }}$ is defined as the number of the bulk MB molecules excited by the laser without Raman enhance effect [19], and $N_{\text {SERS }}$ is the number of MB molecules uniformly spreading on the SERS substrate under the laser spot. Using a $100 \times$ objective lens, the area of the laser spot is around $1 \mu \mathrm{m}^{2}$. Assuming a monolayer of the adsorbed molecules, the value of $N_{\text {SERS }}$ for MB under laser excitation is therefore approximately $5 \times 10^{5}$ molecules [19].

The reusability of a SERS substrate was investigated by repeating the following steps for five cycles: (1) measuring the Raman spectra of an HfN thin film, (2) dropping $10^{-4} \mathrm{M} \mathrm{MB}$ on the surface of the HfN substrate and allowing it to dry in ambient, (3) collecting the Raman spectrum of MB, and (4) cleaning the SERS substrate by ethanol solution and wiping away contaminants from the surface of the HfN thin film.

\section{Results and Discussion}

\subsection{Crystal Orientation of HfN Thin Film}

Figure 2a shows the XRD pattern of samples grown on $\mathrm{Si}(111)$ substrates by using RGT RF magnetron sputtering and conventional mixture gas sputtering techniques, respectively. The thicknesses of samples were approximately $100 \mathrm{~nm}$ measured by cross-sectional FESEM, as shown in Figure S1. All samples show clear peaks at $2 \theta=34.414$ and 39.947 degrees, corresponding to a face-centered cubic (FCC) structure of HfN(111) and (200) planes (Joint Committee on Powder Diffraction Standards (JCPDS) file no. 01-070-282). The additional peak at $2 \theta=57.774$ corresponding to an $\operatorname{HfN}(220)$ plane was found in the samples RGT5, RGT7, and MIX. When the gas-timing $\mathrm{N}_{2}$ turn-on time is longer, the energy per sputtered atom increases, resulting in an increase in crystallinity. Moreover, peak positions of $\mathrm{HfN}(111)$ and $\mathrm{HfN}(200)$ were shifted to higher $2 \theta$ when the gas-timing $\mathrm{N}_{2}$ turnon time increased (as shown in Table S2). During the sputtering process, the total energy of sputtered atoms directly correlates with RF power and working pressure, which could be attributed to the energy transferred by $\mathrm{Ar}+$ to an atom on a target surface (i.e., energy per atom). Since an abrupt Ar turn-off sequence can be interpreted as a decrease in the working pressure, the higher energy of sputtered atoms can be increased with a longer Ar turn-off sequence $[19,20]$. The bias at the target was measured. The absolute value of the difference of the measured bias at the target between $\mathrm{N}_{2}$ turn-on timing and turn-off timing $\left(\mid V_{\mathrm{N}_{2}}\right.$ turn on $\left.-V_{\mathrm{N}_{2} \text { turn off }} \mid\right)$ increased with the $\mathrm{N}_{2}$ turn-on timing, as shown in Figure S2. It can be seen that the absolute value of the self-bias voltage increased when $\mathrm{N}_{2}$ turned on, implying that the energy of sputtered atom was powerfully enhanced. 

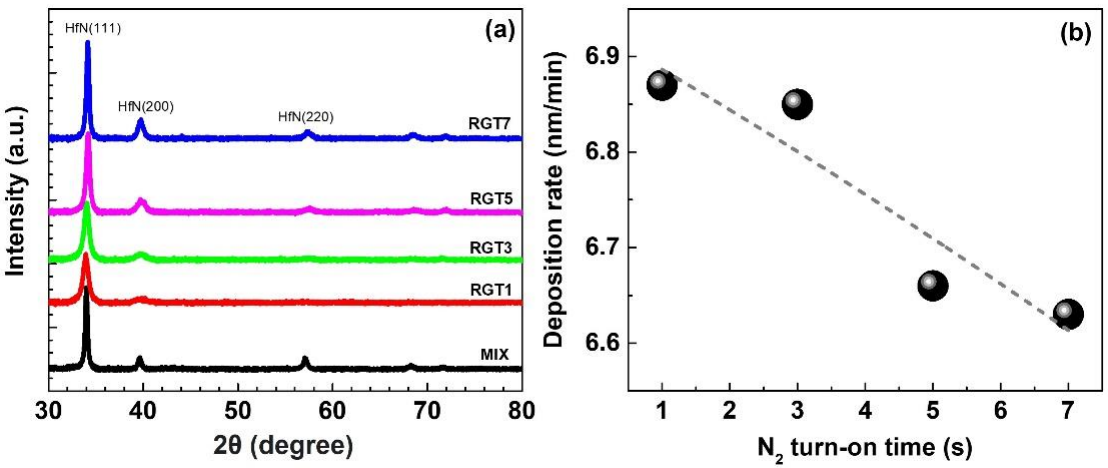

Figure 2. (a) XRD pattern of HfN thin films and (b) deposition rate of HfN thin films.

When a high-energy sputtered atom impinges with other atoms on the substrate, compressive stress is generated, resulting in a peak shift. A similar phenomenon was confirmed in the silver thin films grown via gas-timing RF magnetron sputtering [19]. Figure $2 \mathrm{~b}$ shows the deposition rate of HfN thin films. The deposition rate of HfN via RGT technique gradually decreased from 6.63 to $6.87 \mathrm{~nm} / \mathrm{min}$ when the gas-timing $\mathrm{N}_{2}$ turn-on time increased from 1 to $7 \mathrm{~s}$. Additionally, compared with the conventional mixture gases technique, the deposition rate of the RGT technique is approximately 2.3 times higher owing to the suppression of nitridation on the target surface. The crystallite size was calculated based on the Debye-Scherrer equation. The crystallite sizes of MIX, RGT1, RGT3, RGT5, and RGT7 are 14, 6, 7, 10, and $12 \mathrm{~nm}$, respectively.

\subsection{Morphology of HfN Thin Film}

Figure 3a-c shows AFM images of an HfN thin film deposited by conventional mixture gases and RGT techniques. The MIX sample shows a grain size of approximately $26 \mathrm{~nm}$, while the RGT samples show a smaller range of grain size of approximately $15-19 \mathrm{~nm}$ with a roughness of approximately $0.65-0.93 \mathrm{~nm}$, as shown in Figure 3f. The formation of a small grain size by RGT technique is attributed to an atomic peening effect. Compared with conventional technique, the energy of sputtered atoms is powerfully enriched during the RGT process. Therefore, the sputtered atoms impinged on the surface of thin films possess the sufficient kinetic energy to enhance the mobility of sputtered atoms condensed at the film surface $[19,20]$.

MIX

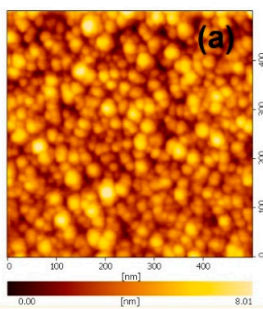

RGT5

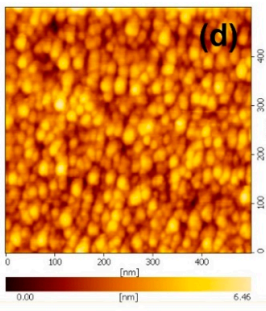

RGT1

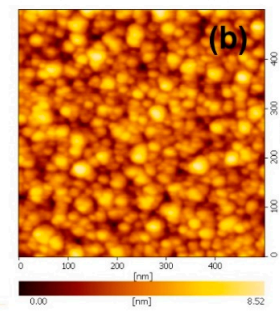

RGT7

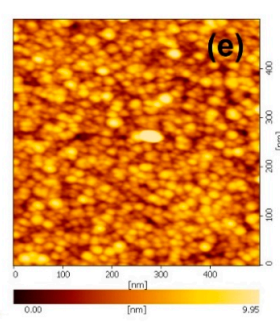

RGT3
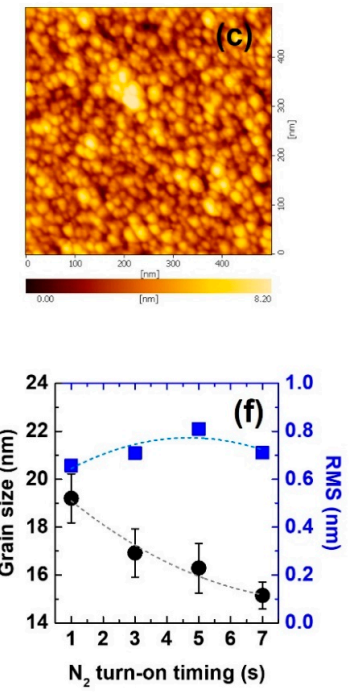

Figure 3. (a-e) AFM images of MIX, RGT1, RGT3, RGT5, and RGT7, respectively. (f) Relationship between the grain size and gas-timing $\mathrm{N}_{2}$ turn-on time (black circles) and the roughness and gastiming $\mathrm{N}_{2}$ turn-on time (blue squares). 


\subsection{Chemical Composition of HfN Thin Film}

Figure 4 shows the Raman spectra of HfN thin films. The MIX sample exhibited the first-order acoustic Raman band at $120-168 \mathrm{~cm}^{-1}$, the first-order optical peak at $520 \mathrm{~cm}^{-1}$, and the shoulder optical band at $685 \mathrm{~cm}^{-1}$ corresponding to HfN bonding [23,24], while the RGT samples showed similar spectra but a blue shift of the first-order acoustic Raman band to $136-168 \mathrm{~cm}^{-1}$, indicating that the MIX sample was nitrogen rich and RGT samples were nonstoichiometry $\mathrm{HfN}_{\mathrm{x}}$ [23].

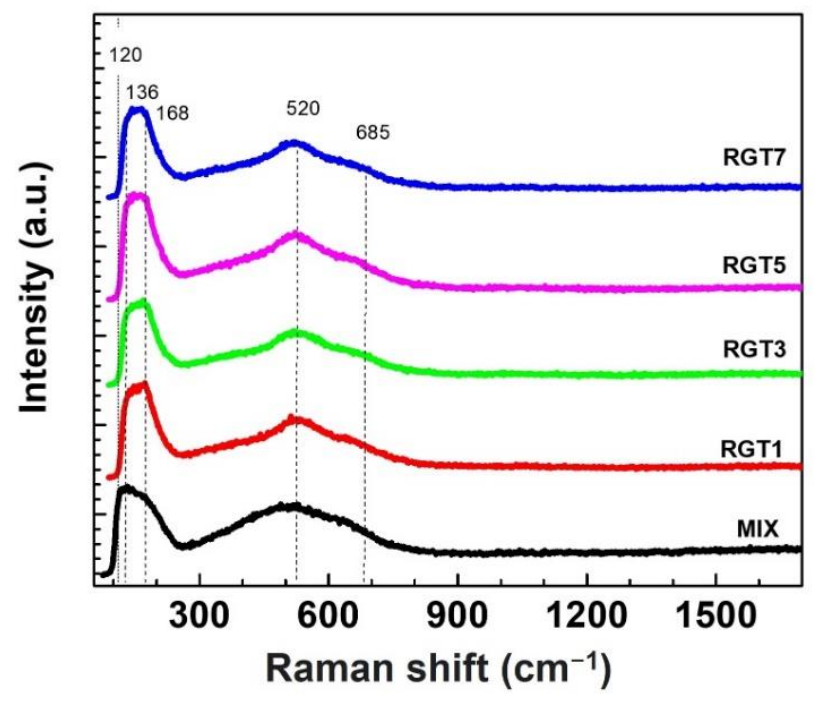

Figure 4. Raman spectra of HfN thin films prepared by RGT and conventional mixture gases techniques.

The chemical composition of the HfN thin films was further determined using XPS analysis. The high-resolution Hf $4 \mathrm{f}$ and N 1s XPS spectra are shown in Figure 5a,b. The XPS spectrum of $\mathrm{Hf} 4 \mathrm{f}$ showed distinguished three peaks at a binding energy of approximately $15.3,17.0$, and $18.5 \mathrm{eV}$, corresponding to Hf-N, O-Hf-N, and Hf-O, respectively [24-26]. The $\mathrm{N} 1 \mathrm{~s}$ XPS spectra of the thin films showed two distinguished peaks at binding energies of 396.2 and $399.5 \mathrm{eV}$ assigned to be Hf-N and chemisorbed nitrogen atoms ( $\mathrm{N}$ abs.) on the surface, respectively $[27,28]$. When the gas-timing $\mathrm{N}_{2}$ turn-on time increased, the intensity and area of the $396.4 \mathrm{eV}$ peak increased, implying an increase in Hf- $\mathrm{N}$ bonding. The sample prepared by conventional mixture gases technique showed a relatively high peak intensity at $399.5 \mathrm{eV}$, implying that nitrogen was absorbed on the surface and could not bind with hafnium atoms (as shown in Figure S3). In contrast, the thin films formed by RGT technique showed higher Hf-N/N abs. ratio, indicating a high ratio of Hf-N bonding due to the high energy per sputtered atom. The $\frac{\mathrm{N}}{\mathrm{Hf}}$ ratio was calculated based on the following equation:

$$
\frac{\mathrm{N}}{\mathrm{Hf}} \text { ratio }=\frac{\left(\frac{\mathrm{A}_{\mathrm{N}-\mathrm{Hf}}+\mathrm{A}_{\mathrm{N}-\mathrm{Hf}-\mathrm{O}}}{1.8}\right)}{\left(\frac{\mathrm{A}_{\mathrm{Hf}}}{7.52}\right)}
$$

where $A_{\mathrm{N}-\mathrm{Hf}}, A_{\mathrm{N}-\mathrm{Hf}-\mathrm{O}}$, and $A_{\mathrm{Hf}}$ are peak areas of N-Hf of N $1 \mathrm{~s}, \mathrm{~N}-\mathrm{Hf}-\mathrm{O}$ of N $1 \mathrm{~s}$, and Hf $4 \mathrm{f}$, respectively. The amounts of 1.8 and 7.52 are relative sensitive factors of $\mathrm{N} 1 \mathrm{~s}$ and $\mathrm{Hf} 4 \mathrm{f}$, respectively. Figure $5 \mathrm{c}$ shows that an $\frac{\mathrm{N}}{\mathrm{Hf}}$ ratio of the prepared films increased from 0.24 to 0.28 , when gas-timing $\mathrm{N}_{2}$ turn-on time increased from 1 to $7 \mathrm{~s}$, while the MIX film showed the lowest $\frac{\mathrm{N}}{\mathrm{Hf}}$ ratio of approximately 0.49 . These results show that RGT is a promising technique to control the chemical composition of an HfN film. 

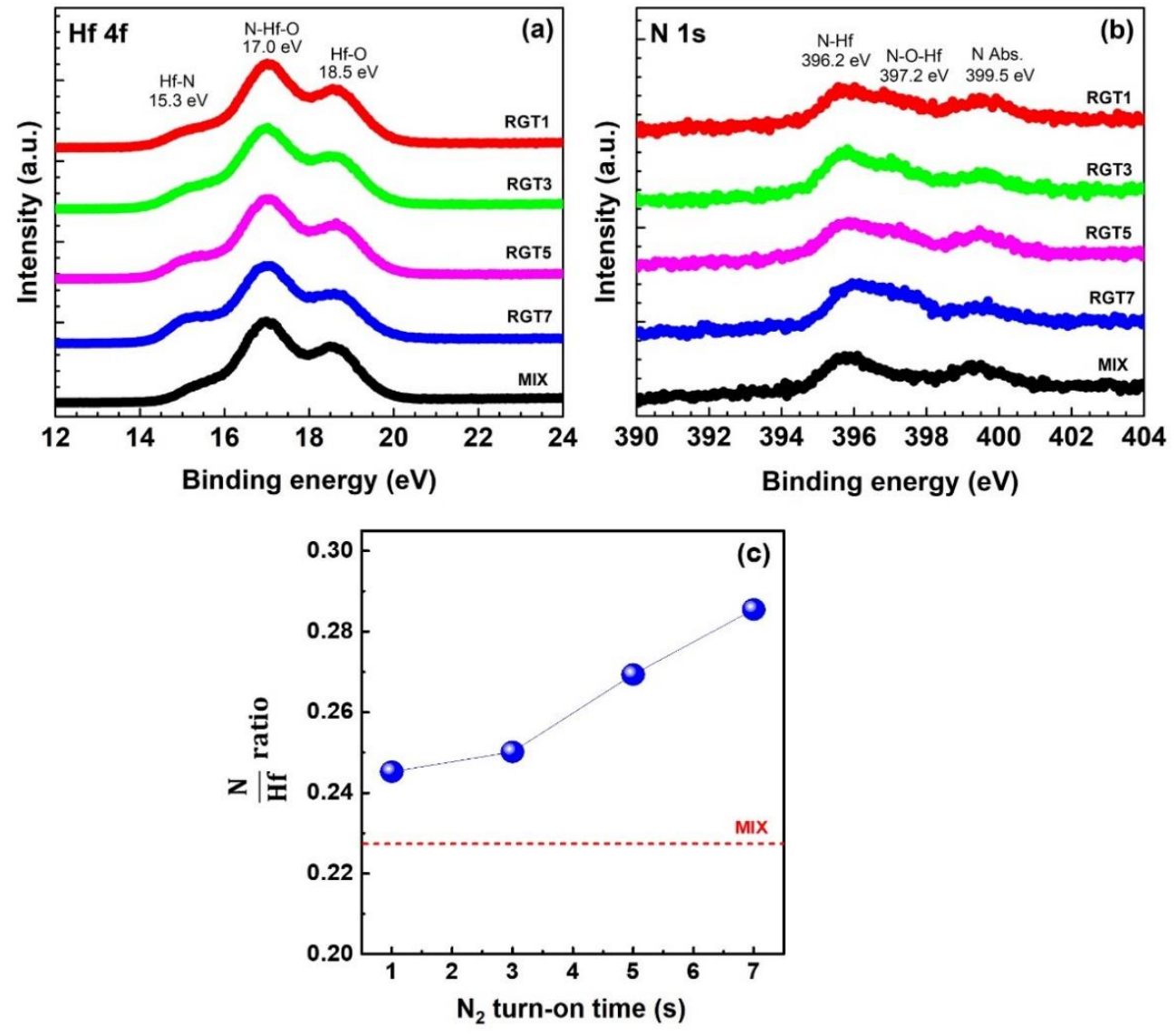

Figure 5. XPS spectra of (a) $\mathrm{Hf} 4 \mathrm{f}$ and (b) $\mathrm{N}$ 1s. (c) Relationship between $\mathrm{N}_{2}$ turn-on time and $\frac{\mathrm{N}}{\mathrm{Hf}}$ ratio.

\subsection{Optical Properties of HfN Thin Film}

The optical absorption spectra of the HfN thin films prepared by different sputtering conditions are shown in Figure 6. All absorbance spectra show similar shapes. The absorption is strong at low wavelength from interband transitions and suddenly decreases to a minimum absorbance of approximately $400 \mathrm{~nm}$ for an $\mathrm{HfN}$ thin film by RGT technique and $543 \mathrm{~nm}$ for an $\mathrm{HfN}$ thienhann film via conventional mixture gases technique. There is absorption at a long wavelength $(\sim 500-900 \mathrm{~nm})$, consistent with previous results [29], demonstrating the characteristics of free electron absorption from $\mathrm{Hf} 4 \mathrm{~d}$ states via intraband transitions [30]. However, the HfN thin films via the conventional mixture gases have a lower absorbance than those synthesized using the RGT technique. This could be due to the difference in chemical compositions of thin films in terms of N/Hf ratio. Additionally, these results demonstrate that the RGT technique is capable of adjusting the number of nitrogen ions in the system, in addition to enhancing the energy reaction formation. As a result of the low energy in the conventional mixture gases technique, the reaction between $\mathrm{Hf}$ and $\mathrm{N}$ is weak, resulting in the chemisorption of nitrogen atoms, as illustrated in Figure $5 b$,c. The longer the gas-timing $\mathrm{N}_{2}$ turn-on time in $\mathrm{HfN}$ films prepared using the RGT technique, the greater the absorbance in the long wavelength region [31]. These optical properties are advantageous for the surface plasmon resonance phenomenon observed in TMN thin films, such as HfN, which can result in a significant enhancement of the electromagnetic mechanism on the surface. 


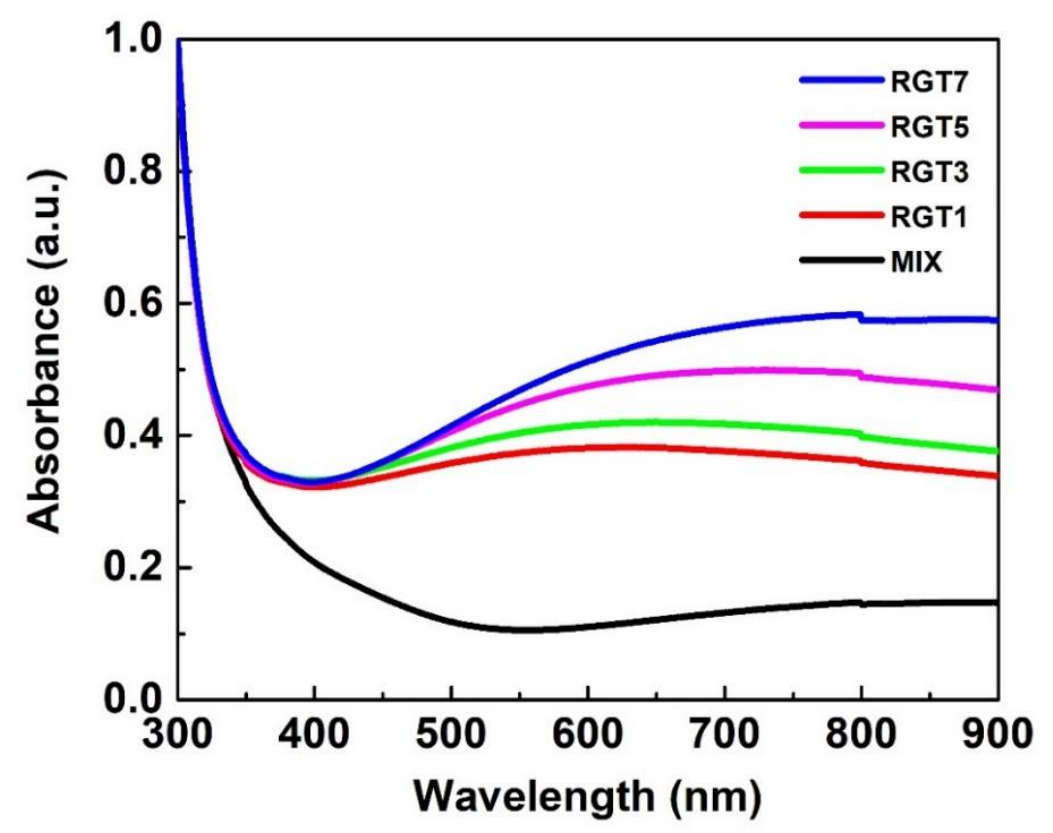

Figure 6. Absorbance spectra of HfN thin films.

\subsection{SERS Performance of HfN Thin Films}

HfN thin films were investigated for their utilization as a SERS substrate by using methylene blue (MB) as the Raman probe molecule. Figure 7a shows Raman spectra of $\mathrm{MB}$ at a concentration of $10^{-4} \mathrm{M}$ on HfN thin films and a bare Si substrate. It can be seen that HfN thin films clearly showed Raman spectra with peaks at approximately 440, 768, 1180,1394 , and $1623 \mathrm{~cm}^{-1}$, corresponding to the skeletal deformation of C-N-C, in-plane bending of $\mathrm{C}-\mathrm{H}$, stretching of $\mathrm{C}-\mathrm{N}$, asymmetrical stretching of $\mathrm{C}-\mathrm{N}$, and ring stretching of C-C, respectively [32]. The Raman intensity of MB molecules obtained from an HfN thin film substrate was five times higher than from a bare Si substrate. These results clearly show that HfN films can be successfully used as a SERS substrate. Comparing HfN thin films and focusing on the intensity of the main peaks of MB at a Raman shift of $1623 \mathrm{~cm}^{-1}$, all HfN thin films prepared by RGT technique showed a higher intensity than that of the conventional mixture gases technique, as shown in Figure $7 \mathrm{~b}$.

An HfN thin film as a SERS substrate can be described similar to other TMN thin films, which are based on two working mechanisms: chemical enhancement mechanism and electromagnetic mechanism. Normally, the chemical enhancement mechanism is contributed by charge transfer between molecules and a SERS substrate. Due to the fact that MB has an excitation peak at approximately $663 \mathrm{~nm}$ [33], this work utilized a laser with an excitation wavelength of $632 \mathrm{~nm}(1.96 \mathrm{eV})$. Meanwhile, the energy band gap of HfN was approximately $3.6 \mathrm{eV}$ calculated from a Tauc plot (as shown in Figure S4), which was significantly greater than the energy of the exciting source. On the other hand, electromagnetic enhancement is a result of optical properties, such as LSPR and surface morphology (roughness and grain size), which can result in a large number of "hot-spots" at nanoscales. As the particle size increases, the particles absorb less light and scatter more through inelastic scattering, which should decrease the overall SERS intensity [34]. Furthermore, the RGT technique provides the controllable chemical composition, affording a thin film with a relatively high absorption at the wavelength of a Raman excitation laser $(632 \mathrm{~nm}, 1.96 \mathrm{eV})$. Regarding the HfN thin films prepared by RGT technique, it was found that the peak intensity gradually increased when the turn-on $\mathrm{N}_{2}$ gas-timing increased. RGT7 showed the highest peak intensity with an EF of approximately $8 \times 10^{4}$ (inset of Figure $7 \mathrm{~b}$ ). As a result, electromagnetic enhancement may play a role in the SERS effect of the HfN thin film. 

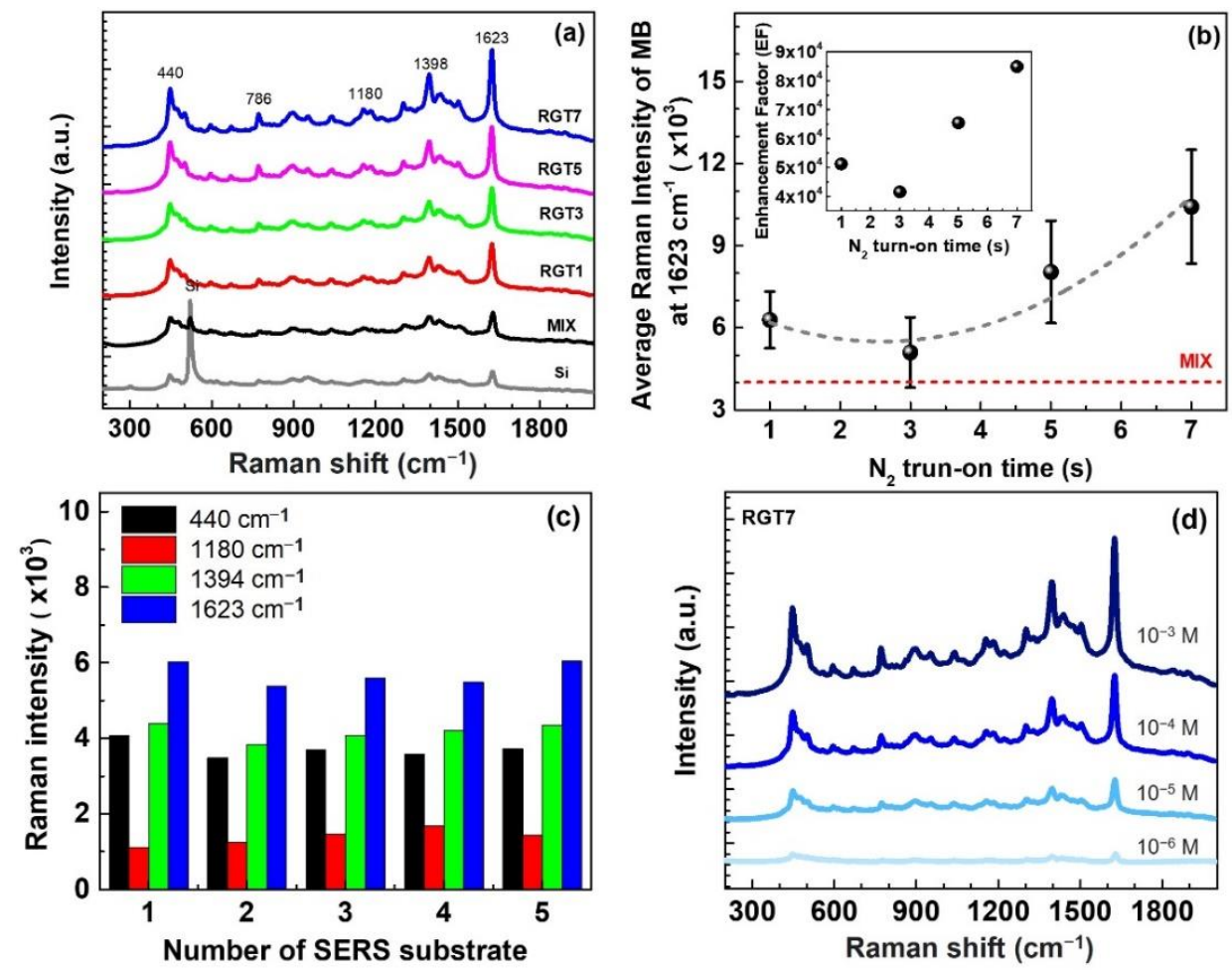

Figure 7. SERS activity of HfN thin films. (a) Raman spectra of MB $10^{-4} \mathrm{M}$ on HfN thin films and a bare Si substrate. (b) Raman intensity of MB at a Raman shift of $1623 \mathrm{~cm}^{-1}$. Inset is enhancement factor value. (c) Reproducibility of RGT7. (d) Raman spectra of MB in the concentration range of $10^{-3}$ down to $10^{-6} \mathrm{M}$ using RGT7 as a SERS substrate.

Reproducibility is one parameter for the practical application of a SERS substrate. Five samples of RGT7 were used to determine the reproducibility of the HfN thin film as a SERS substrate. Raman intensities of MB at Raman shifts of 440,1180, 1394, and $1623 \mathrm{~cm}^{-1}$ of each sample were compared, as shown in Figure 7c. There were no obvious differences among each sample, indicating high reproducibility and uniformity of Raman signal that was provided by RGT technique. The limit of detection (LOD) of RGT7 was investigated by varying $\mathrm{MB}$ concentrations in the range of $10^{-7}$ to $10^{-3} \mathrm{M}$, as shown in Figure $7 \mathrm{~d}$. The Raman signal of $\mathrm{MB}$ was detectable down to a concentration of $10^{-6} \mathrm{M}$.

The reusability of an HfN thin film as a SERS substrate was also investigated by the process, as shown in Figure 8a. The Raman spectra of an HfN thin film were collected repeatedly for five cycles following MB dropping and cleaning, as shown in Figure $8 \mathbf{b}$. After removing MB from the surface of the HfN thin film, the Raman signal of MB vanished, leaving the Raman spectrum identical to that obtained prior to dropping MB (1st-HfN), as indicated by the spectrum labeled as 2nd-HfN. When the MB was dropped again onto the same substrate, a similar Raman signal was observed, as indicated by the spectrum labeled as 2nd-HfN/MB. After five cycles of testing, the Raman signal of MB was still detectable, and the Raman spectrum of 6th-HfN was similar to that of $1 \mathrm{st}-\mathrm{HfN}$, indicating that the HfN thin film can be reused as a SERS substrate. The reusable HfN thin film substrate is made possible by its superior mechanical properties and inherent chemical stability in harsh environments. Compared with Ag- or Au-based nanostructured matrices, the HfN thin film substrate is less sensitive. However, the reusability of the material is a significant advantage for practical application. 

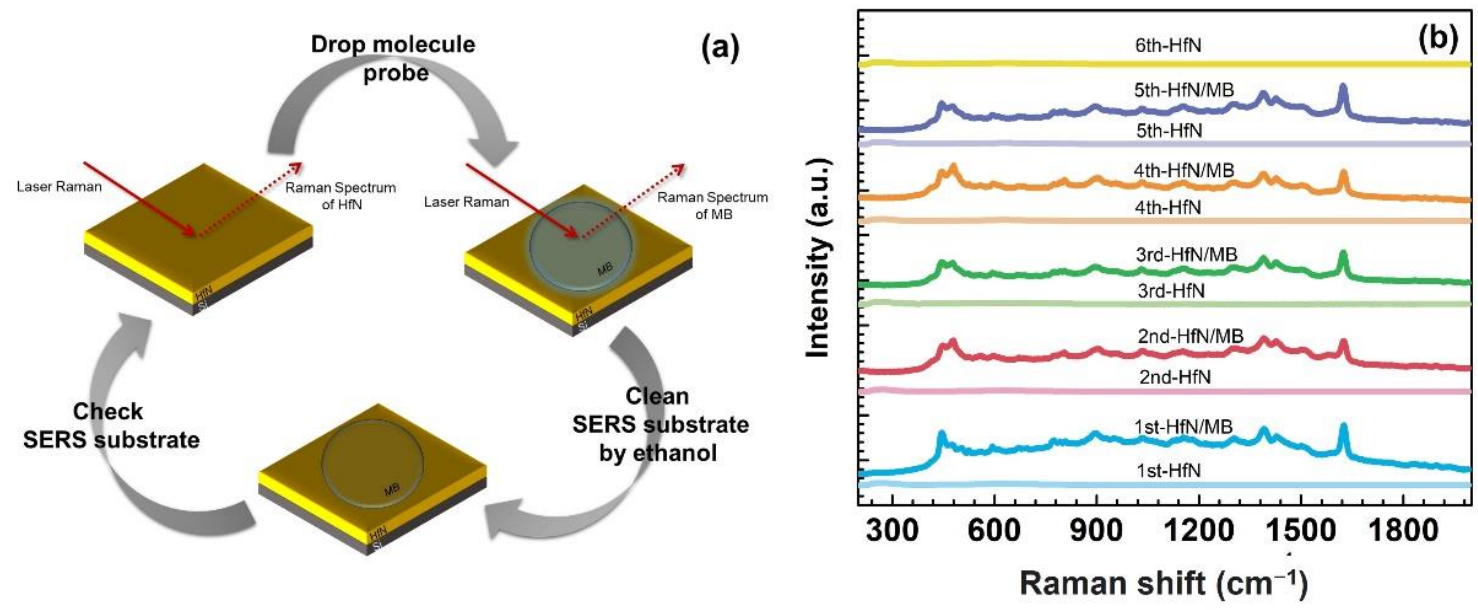

Figure 8. Reusability of HfN thin films. (a) Schematic view of test steps. (b) Raman spectra of HfN thin film and $\mathrm{MB}$.

\section{Conclusions}

HfN thin films with tailored properties for SERS were successfully prepared using RGT RF magnetron sputtering technique. The sequence of gas-timing turn-on $\mathrm{N}_{2}$ has a direct effect on the morphology, chemical composition, and optical absorption properties of HfN thin films. HfN thin films with a grain size of approximately $15 \mathrm{~nm}$ were formed. The crystallinity increased, and the atomic ratios of nitrogen and hafnium can be controlled to 0.65 when the gas-timing $\mathrm{N}_{2}$ turn-on time increased to $7 \mathrm{~s}$. The SERS activity of an HfN thin film was observed using a MB molecule, showing an enhancement factor of $8.5 \times 10^{4}$ and a LOD of $10^{-6} \mathrm{M}$. The advantage of an HfN thin film is that it can be reused as SERS substrates up to five times. A superior performance of HfN as a SERS substrate can be attributed to its tailored grain size and chemical composition, which results in an increase in the hot spot effect.

Supplementary Materials: The following are available online at https://www.mdpi.com/article/10 .3390/cryst12010078/s1: Table S1: Sputtering parameter for HfNx deposited by RGT RF magnetron sputtering; Table S2: Summary of the position of the HfN(111) peak; Figure S1: Cross-sectional FE-SEM images of HfN thin films; Figure S2: Absolute value of the difference in self-bias voltage as a function of $\mathrm{N}_{2}$ turn-on timing; Figure S3: (a) Deconvoluted spectra of $\mathrm{N} 1 \mathrm{~s}$ and (b) N-Hf/N abs. ratio of an HfN thin film prepared at different sputtering conditions; Figure S4: Tauc plots of RGT7.

Author Contributions: Methodology, N.S. (Nguentra Sucheewa), W.A., N.S. (Nataporn Sowasod), T.P., S.K., M.O., M.F., K.T., T.L. and T.W.; resources, J.N., A.K., W.T. and T.L.; data curation, N.S. (Nataporn Sowasod) and W.W.; writing—original draft preparation, N.S. (Nguentra Sucheewa); writing-review and editing, W.W.; supervision, W.W., A.K., W.T. and J.N.; project administration, W.W., A.K. and J.N.; funding acquisition, W.W. and A.K. All authors have read and agreed to the published version of the manuscript.

Funding: National Research Council of Thailand (NRCT) (2564NRCT322246). National Science and Technology Development Agency (NSTDA) (P2150364).

Data Availability Statement: The data presented in this study are available within the article.

Acknowledgments: The authors gratefully acknowledge the facility support from the Nano-Characterization Research Laboratory, National Nanotechnology Center (NANOTEC), Nanotechnology and Material Analytical Instrument Service Unit (NMIS), and College of Materials Innovation and Technology, King Mongkut's Institute of Technology Ladkrabang. Nguentra Sucheewa acknowledges the financial support from the National Research Council of Thailand (NRCT) (2564NRCT322246). This work was partially supported by the National Science and Technology Development Agency (NSTDA), Thailand, through grant P2150364.

Conflicts of Interest: The authors declare no conflict of interest. 


\section{References}

1. Sharma, B.; Frontiera, R.R.; Henry, A.-I.; Ringe, E.; Van Duyne, R.P. SERS: Materials, applications, and the future. Mater. Today 2012, 15, 16-25. [CrossRef]

2. Cong, S.; Wang, Z.; Gong, W.; Chen, Z.; Lu, W.; Lombardi, J.R.; Zhao, Z. Electrochromic semiconductors as colorimetric SERS substrates with high reproducibility and renewability. Nat. Commun. 2019, 10, 678. [CrossRef] [PubMed]

3. Aroca, R. Surface-Enhanced Vibrational Spectroscopy; John Wiley \& Sons: Chichester, UK, 2006; pp. 107-132.

4. Moskovits, M. Persistent misconceptions regarding SERS. Phys. Chem. Chem. Phys. 2013, 15, 5301-5311. [CrossRef]

5. Sinthiptharakoon, K.; Sapcharoenkun, C.; Nuntawong, N.; Duong, B.; Wutikhun, T.; Treetong, A.; Meemuk, B.; Kasamechonchung, P.; Klamchuen, A. Conductive scanning probe microscopy of the semicontinuous gold film and its SERS enhancement toward two-step photo-induced charge transfer and effect of the supportive layer. Appl. Surf. Sci. 2018, 441, 364-371. [CrossRef]

6. Wei, H.; Wu, M.; Dong, Z.; Chen, Y.; Bu, J.; Lin, J.; Yu, Y.; Wei, Y.; Cui, Y.; Wang, R. Composition, microstructure and SERS properties of titanium nitride thin film prepared via nitridation of sol-gel derived titania thin films. J. Raman Spectrosc. 2017, 48, 578-585. [CrossRef]

7. Zhao, F.; Xue, X.; Fu, W.; Liu, Y.; Ling, Y.; Zhang, Z. TiN Nanorods as Effective Substrate for Surface-Enhanced Raman Scattering J. Phys. Chem. C 2019, 123, 29353-29359. [CrossRef]

8. Kumar, M.; Umezawa, N.; Ishii, S.; Nagao, T. Examining the Performance of Refractory Conductive Ceramics as Plasmonic Materials: A Theoretical Approach. ACS Photonics 2016, 3, 43-50. [CrossRef]

9. Guo, W.-P.; Mishra, R.; Cheng, C.-W.; Wu, B.-H.; Chen, L.-J.; Lin, M.-T.; Gwo, S. Titanium Nitride Epitaxial Films as a Plasmonic Material Platform: Alternative to Gold. ACS Photonics 2019, 6, 1848-1854. [CrossRef]

10. Exarhos, S.; Alvarez-Barragan, A.; Aytan, E.; Balandin, A.A.; Mangolini, L. Plasmonic Core-Shell Zirconium Nitride-Silicon Oxynitride Nanoparticles. ACS Energy Lett. 2018, 3, 2349-2356. [CrossRef]

11. O'Neill, D.B.; Frehan, S.K.; Zhu, K.; Zoethout, E.; Mul, G.; Garnett, E.C.; Huijser, A.; Askes, S.H.C. Ultrafast Photoinduced Heat Generation by Plasmonic HfN Nanoparticles. Adv. Opt. Mater. 2021, 9, 2100510. [CrossRef]

12. Patsalas, P.; Kalfagiannis, N.; Kassavetis, S.; Abadias, G.; Bellas, D.V.; Lekka, C.; Lidorikis, E. Conductive nitrides: Growth principles, optical and electronic properties, and their perspectives in photonics and plasmonics. Mater. Sci. Eng. R Rep. 2018, 123, 1-55. [CrossRef]

13. Hu, C.; Gu, Z.; Wang, J.; Zhang, K.; Zhang, X.; Li, M.; Zhang, S.; Fan, X.; Zheng, W. Nature of Tunable Optical Reflectivity of Rocksalt Hafnium Nitride Films. J. Phys. Chem. C 2014, 118, 20511-20520. [CrossRef]

14. Heo, S.-B.; Lee, H.-M.; Kim, D.-I.; Choi, D.-H.; Lee, B.-H.; Kim, M.-G.; Lee, J.-H. Effect of Substrate Bias Voltage on the Properties of Hafnium Nitride Films Deposited by Radio Frequency Magnetron Sputtering Assisted by Inductive Coupled Nitrogen Plasma. Trans. Electr. Electron. Mater. 2011, 12, 209-212. [CrossRef]

15. Thorsteinsson, D.; Gudmundsson, J.T. Growth of HfN thin films by reactive high power impulse magnetron sputtering. AIP Adv. 2018, 8, 035124. [CrossRef]

16. Phae-Ngam, W.; Prathumsit, J.; Gitgeatpong, G.; Chananonnawathorn, C.; Lertvanithphol, T.; Saekow, B.; Nakajima, H.; Horprathum, M. Effect of post annealed treatment on HfN thin films prepared by DC reactive magnetron sputtering. AIP Conf. Proc. 2020, 2279, 120002. [CrossRef]

17. Chun, S.-Y. Characteristics of HfN coatings by inductively coupled plasma-assisted magnetron sputtering. J. Korean Ceram. Soc. 2021, 58, 178-183. [CrossRef]

18. Khemasiri, N.; Paleeya, N.; Phromyothin, D.S.; Horprathum, M.; Porntheeraphat, S.; Sungthong, A.; Nukeaw, J.; Pratontep, S Silicon Nitride Thin Films Deposited By Reactive Gas-Timing Magnetron Sputtering For Protective Coating Applications. Adv. Mater. Lett. 2015, 6, 554-559. [CrossRef]

19. Ukahapunyakulab, P.; Gridsadanurakab, N.; Sapcharoenkunc, C.; Treetongc, A.; Kasamechonchungc, P.; Khemthongc, P.; Horprathumd, M.; Porntheeraphatd, S.; Wongwiriyapane, W.; Nukeawe, J.; et al. Texture orientation of silver thin films grown via gas-timing radio frequency magnetron sputtering and their SERS activity. RSC Adv. 2016, 9, 7661-7667. [CrossRef]

20. Khemasiri, N.; Chananonnawathorn, C.; Klamchuen, A.; Jessadaluk, S.; Pankiew, A.; Vuttivong, S.; Eiamchai, P.; Horprathum, M.; Pornthreeraphat, S.; Kasamechonchung, P.; et al. Crucial role of reactive pulse-gas on a sputtered $\mathrm{Zn}_{3} \mathrm{~N}_{2}$ thin film formation. RSC Adv. 2016, 6, 94905-94910. [CrossRef]

21. Lertvanithphol, T.; Rakreungdet, W.; Chananonnawathorn, C.; Eiamchai, P.; Limwichean, S.; Nuntawong, N.; Patthanasettakul, V.; Klamchuen, A.; Khemasiri, N.; Nukeaw, J.; et al. Spectroscopic study on amorphous tantalum oxynitride thin films prepared by reactive gas-timing RF magnetron sputtering. Appl. Surf. Sci. 2019, 492, 99-107. [CrossRef]

22. He, G.; Meng, G.W.; Zhang, L.D.; Liu, M. Temperature-Dependent interfacial chemical bonding states and band alignment of HfOxNy/SiO $/ 2$ Si gate stacks. Appl. Phys. Lett. 2007, 91, 232910. [CrossRef]

23. Stoehr, M.; Seo, H.-S.; Petrov, I.; Greene, J.E. Effect of off stoichiometry on Raman scattering from epitaxial and polycrystalline HfNx $(0.85 \leq x \leq 1.50)$ grown on $\mathrm{MgO}(001)$. J. Appl. Phys. 2008, 104, 033507. [CrossRef]

24. Zhang, D.; Qi, Z.; Wei, B.; Shen, H.; Wang, Z. Microstructure and corrosion behaviors of conductive Hf/HfN multilayer coatings on magnesium alloys. Ceram. Int. 2018, 44, 9958-9966. [CrossRef]

25. Piallat, F.; Beugin, V.; Gassilloud, R.; Dussault, L.; Pelissier, B.; Leroux, C.; Caubet, P.; Vallée, C. Interface and plasma damage analysis of PEALD TaCN deposited on $\mathrm{HfO}_{2}$ for advanced CMOS studied by angle resolved XPS and C-V. Appl. Surf. Sci. 2014, 303, 388-392. [CrossRef] 
26. Egorov, K.V.; Lebedinskii, Y.Y.; Soloviev, A.A.; Chouprik, A.A.; Azarov, A.Y.; Markeev, A.M. Initial and steady-state Ru growth by atomic layer deposition studied by in situ Angle Resolved X-ray Photoelectron Spectroscopy. Appl. Surf. Sci. 2017, 419, 107-113. [CrossRef]

27. Caicedo, J.; Mozafari, M.; Aperador, W. Determination of superlattice effect on metal-ceramic nano-structures. Results Phys. 2015, 5, 241-249. [CrossRef]

28. Phadke, P.; Sturm, J.M.; van de Kruijs, R.W.; Bijkerk, F. Sputtering and nitridation of transition metal surfaces under low energy, steady state nitrogen ion bombardment. Appl. Surf. Sci. 2020, 505, 144529. [CrossRef]

29. Chung, S.; Wen, X.; Huang, S.; Gupta, N.; Conibeer, G.; Shrestha, S.; Harada, T.; Kee, T.W. Nanosecond long excited state lifetimes observed in hafnium nitride. Sol. Energy Mater. Sol. Cells 2018, 169, 13-18. [CrossRef]

30. Wang, J.; Li, F.; Ao, J.; Zhong, Y.; Chen, Z.Q. First Principle Study of the Optical Properties of Transition Metal Nitrides XN (X = Ti, Zr, Hf). Adv. Mater. Res. 2013, 668, 710-714. [CrossRef]

31. Du, M.; Shen, Z. Enhanced and tunable double Fano resonances in plasmonic metasurfaces with nanoring dimers. J. Phys. D Appl. Phys. 2020, 54, 145106. [CrossRef]

32. Zannotti, M.; Rossi, A.; Giovannetti, R. SERS Activity of Silver Nanosphere, Triangular Nanoplates, Hexagonal Nanoplates and Quasi-Spherical Nanoparticles: Effect of Shape and Morphology. Coatings 2020, 10, 288. [CrossRef]

33. Pahang, F.; Parvin, P.; Ghafoorifard, H.; Bavali, A.; Moafi, A. Fluorescence properties of methylene blue molecules coupled with metal oxide nanoparticles. OSA Contin. 2020, 3, 688. [CrossRef]

34. Cao, Y.; Zhang, J.; Yang, Y.; Huang, Z.; Long, N.V.; Fu, C. Engineering of SERS Substrates Based on Noble Metal Nanomaterials for Chemical and Biomedical Applications. Appl. Spectrosc. Rev. 2015, 50, 499-525. [CrossRef] 